This item was submitted to Loughborough's Research Repository by the author.

Items in Figshare are protected by copyright, with all rights reserved, unless otherwise indicated.

\title{
Simulation methodology for synthesis of antenna substrates with microscale inclusions
}

PLEASE CITE THE PUBLISHED VERSION

http://dx.doi.org/10.1109/TAP.2012.2189736

PUBLISHER

(C) IEEE

VERSION

AM (Accepted Manuscript)

LICENCE

CC BY-NC-ND 4.0

REPOSITORY RECORD

Njoku, Chinwe C., W.G. Whittow, and J.C. Vardaxoglou. 2019. "Simulation Methodology for Synthesis of Antenna Substrates with Microscale Inclusions”. figshare. https://hdl.handle.net/2134/9993. 
This item was submitted to Loughborough's Institutional Repository (https://dspace.lboro.ac.uk/) by the author and is made available under the following Creative Commons Licence conditions.

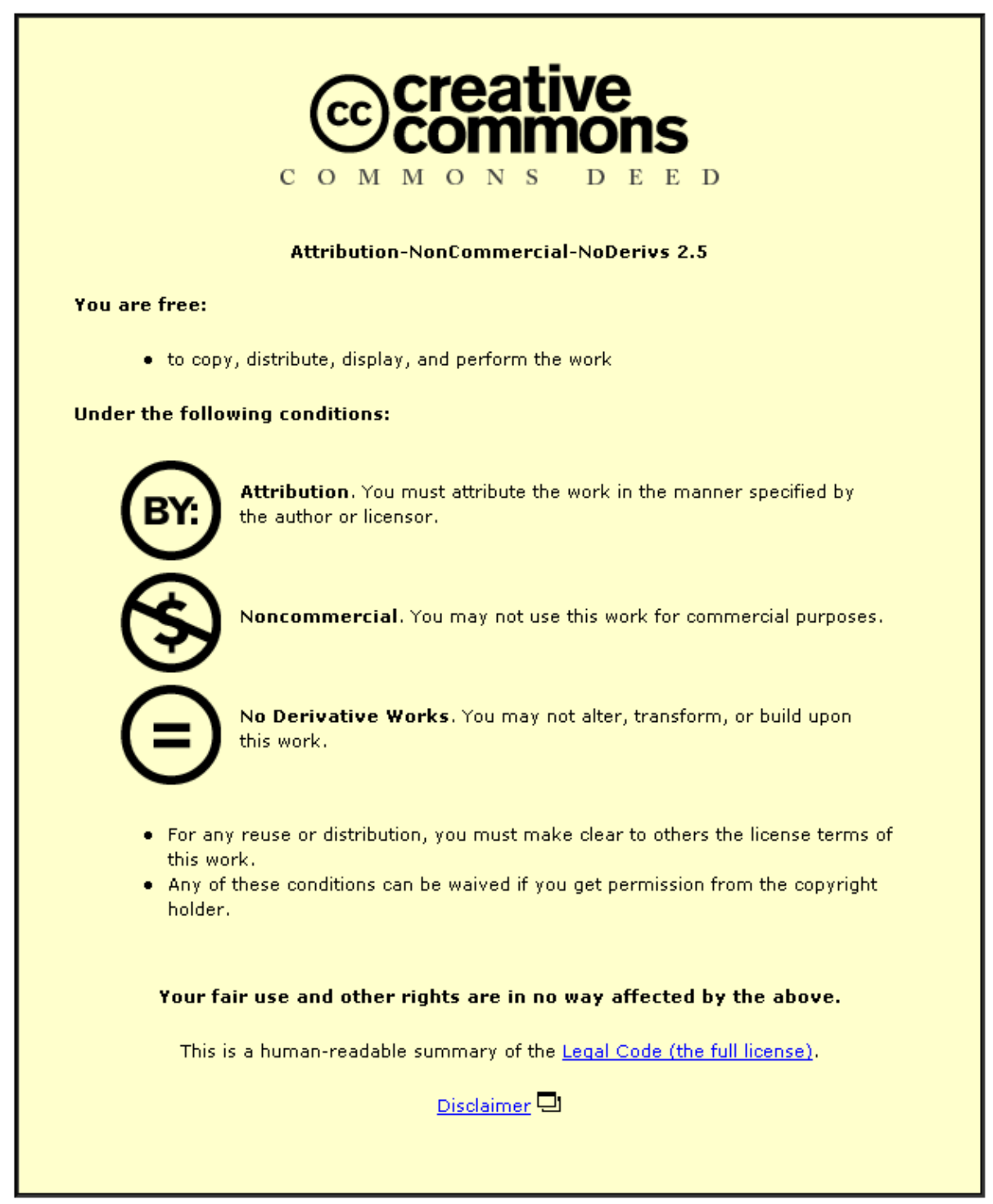

For the full text of this licence, please go to: http://creativecommons.org/licenses/by-nc-nd/2.5/ 


\title{
Simulation Methodology for Synthesis of Antenna Substrates with Micro-scale Inclusions
}

\author{
Chinwe C. Njoku, William G. Whittow, and J(Yiannis) C. Vardaxoglou, Senior Member, IEEE
}

\begin{abstract}
This paper investigates the behaviour of heterogeneous dielectric substrates synthesised by adding microsized inclusions to a host medium. The paper compares different analytical formulations for small spherical inclusions in infinite media and assesses their suitability to be applied to different geometries. The effective permittivity of the mixture has been shown to be dependent on the properties of the host and inclusions' materials as well as the size and spacing of the spheres. Electromagnetic (EM) simulations using a plane wave excitation have also been carried out investigating thin (finite) layers of both spherical and cubical inclusions. A rectification algorithm was employed to correctly obtain the effective permittivity from the scattering parameters. The analytical results of the infinite medium showed good agreement with the EM simulations of our samples of finite thickness. Finally, a patch antenna with a heterogeneous substrate has been designed to show that the method can be applied to a finite geometry which is finite in three dimensions.
\end{abstract}

Index Terms-Heterogeneous dielectric substrates, heterogeneous mixtures, permittivity, rectification algorithm.

\section{INTRODUCTION}

Nanomaterials have inherent advantages due to their high surface area to volume ratio including increased hardness, improved physical strength and electrical conductivity [1], [2]. These material advantages have led to a multibillion dollar industry where the technology and possible structures are developing at a rapid rate. This new technology can potentially control the placement of individual dielectric and metallic particles (or conducting carbon nanotubes) which can be used to design new antenna systems that have fabricational (time, environmental and cost savings), physical, as well as electromagnetic advantages over conventional methods. For example, conventional patch antennas are made using destructive processes where a copper layer covers the entire surface and then environmentally damaging chemicals are used to etch away the unwanted sections. By using additive manufacturing processes such as electrodeposition and selfassembly [3-5] complete antenna structures and associated RF components including the feed and the balun can be

Manuscript received July 7, 2011. This work was supported by EPSRC under Grant No. EP/I01490X/1. The authors are with the Wireless Communications Research Group in the School of Electronic, Electrical and Systems Engineering, Loughborough University, Loughborough, LE11 3TU, UK (e-mail: j.c.vardaxoglou@lboro.ac.uk). envisioned, see, for example, Fig. 1. By integrating the fabrication of the antenna, substrate and RF circuitry into one process, there are potential time and cost savings within the design process, resulting in manufactured products with smaller physical and EM variations. As material, labour and environmental costs increase and the cost of nanotechnology decreases, this technique of fabricating antennas may become cost effective especially for high permittivity substrates which are often prohibitively expensive. Physical advantages are realised as a scratch resistance radome with increased physical durability can be incorporated into the same fabrication process.

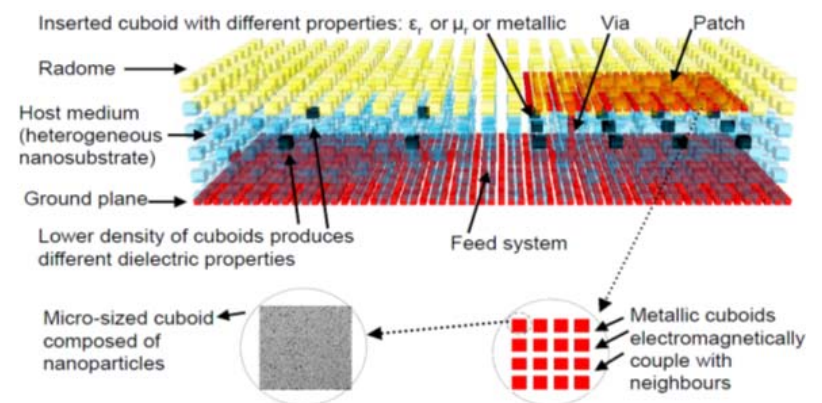

Fig. 1. A sketch showing an antenna system with a heterogeneous substrate (not to scale).

The aim of this work is to investigate the feasibility of designing and fabricating novel antenna integrated systems with heterogeneous substrates, by using micro-sized cuboids composed of nanomaterials. In the case of the particles fused in a nano construction way then these could be classed as nanomaterial antennas. There are also numerous EM benefits. It has been known for a long time that the effective permittivity, permeability and losses of a material can be theoretically controlled by adding inclusions with distinct properties in a cubic lattice [6-15]. This research includes metamaterials [10], artificial materials [12] and double negative materials [14], [15]. Therefore, by enabling heterogeneous substrates, the antenna designer will have two new degrees of freedom compared to conventional substrates: 1) the substrate height and 2) the EM properties and these can be used to design bespoke substrates for antenna systems that must be incorporated into increasingly compact packaging. In previous work, the authors have shown that a highly efficient, compact antenna with a large bandwidth can be designed with a thus far unrealisable substrate which has equality of permittivity and permeability [16]. The heterogeneous 
mixtures described in this paper may enable such materials. Textured dielectrics have been used to reduce the size of the antenna while not adversely affecting the bandwidth or efficiency by using a lower permittivity in areas with high current densities and a higher permittivity elsewhere [17]. The author proposed further improvements could be obtained by using a smooth variation between different dielectric regions. In this work, this could be achieved by controlling the volume ratio of the inclusions as shown in Fig. 1, whereby the permittivity can be designed to have a continuous variation both horizontally and also vertically. In addition, by carefully placing the conducting inclusions, electromagnetic bandgap (EBG) structures can be incorporated into the system to control unwanted current flow.

The purpose of this paper is to investigate heterogeneous media in the above context using 3D Finite-Difference TimeDomain (FDTD) simulations using EMPIRE XCcel'TM, with initial emphasis on their use as substrates in microwave antennas. The results shown for effective permittivity, $\varepsilon_{\text {eff }}$ and permeability, $\mu_{e f f}$ in this paper are the relative values. The interactions between particles on an atomic or quantum level have not been considered. In the FDTD simulations, the host material and the inclusions are assumed to be lossless and have non-dispersive EM properties. In this paper, the term 'heterogeneous' refers to a medium within which elements of different EM properties have been included in a cubic lattice. In Section II, there is a brief account of relevant theoretical equations from reviewed literature on the effective EM properties of infinite heterogeneous media, which is the theoretical foundation which underpins this research. In Section III, we present a "resonant inverse scattering" method [18] of extracting the $\varepsilon_{e f f}$ and $\mu_{\text {eff }}$ of a material from the simulated scattering (S-) parameters. The EM method is validated by inputting the S-parameters from the simulation of a dielectric slab of known permittivity into the extraction process. Simulations and analysis of heterogeneous media using cubic and spherical inclusions are then carried out in Section IV. The results of these simulations are compared with those from the equations by [6], [11], [14]. Parametric studies of the main variables on the effective $\varepsilon$ and $\mu$ of the media are carried out. The work is extended from an infinite to a finite media and is applied to a patch antenna design in Section V. Finally, conclusions are made in Section VI.

The key novel contributions of this paper include: introducing the concept of synthesising microwave antennas using nanomaterials; using spherical and cubical inclusions and applying FDTD EM simulations with a suitable algorithm to find the effective permittivity of heterogeneous substrates of finite volumes, - on which antennas and other circuit components can be printed.

\section{THEORETICAL ANALYSIS}

The work in [6] forms the basis into the EM possibilities with heterogeneous mixtures, although the earliest work on heterogeneous media is that shown in [7]. These papers assume that the inclusions are much smaller than the wavelength but there are slight variations and some do not contain frequency terms or the properties of the host.

In [6], the geometry used was a cubic array of spherical inclusions in a semi-infinite homogenous medium, on which a uniform plane wave impinges. Note, in this work the term "particle" defines the inclusion/element and is not an individual nanoparticle. By describing the total electric (E) and magnetic $(\mathrm{H})$ fields within the medium as a sum of the individual incident fields and the scattered fields from each sphere, the effective EM properties were obtained.

The Clausius-Mossotti (C-M) equation given in (1) has been used for different effective medium theories and is presented differently in [9-14]. Cubic lattices have been used for simplicity but other lattices are also possible [19].

Reference [10] states the C-M equation using the full scattering coefficients of a sphere from [20]. But its equations do not account for the host's EM properties $\left(\varepsilon_{1}\right.$ and $\left.\mu_{1}\right)$ and may not give suitable results if the host's values differ from unity. A similar analysis in [11] includes $\varepsilon_{1}$ and $\mu_{1}$. Reference [12] is relevant to structures with conducting inclusions. However, it does not have a frequency term, uses an interaction constant dependent on the particle's spacing and polarisabilities dependent on the particle's shape. Equations in [13] are similar to those in [6] but do not have a frequency term. In [13] the particle's EM properties $\left(\varepsilon_{2}\right.$ and $\left.\mu_{2}\right)$ are assumed the same as its bulk value, while other authors determine them to be functions of the particle's size, frequency and bulk EM values. Table I summarises the symbols used for the parameters in this paper.

A general mathematical expression for the effective EM properties of particle-embedded mixtures [9] is given in (1) as

$$
K_{\mathrm{eff}}=K_{1} \frac{\left[\left(K_{2}+2 K_{1}\right)\left(K_{1}-K_{2}\right)^{-1}\right]-2 p+C\left(K_{1}, K_{2}, p\right)}{\left[\left(K_{2}+2 K_{1}\right)\left(K_{1}-K_{2}\right)^{-1}\right]+p+C\left(K_{1}, K_{2}, p\right)}
$$

where $K_{1}, K_{2}$ and $K_{\text {eff }}$ are the appropriate parameters $(\varepsilon, \mu, \sigma)$ of the host medium, the inclusions and the mixture respectively; $p$ is the total volume fraction of the inclusions, based on the local unit cell of the medium. $C\left(K_{1}, K_{2}, p\right)$ represents corrections for higher-order multipole terms as a result of the decomposition of the scattered field [6], [20].

A modification to the equations in [14] differentiates the inclusions' densities and polarisabilities in electric and magnetic modes. This is an adoption of the equations in [13] which looked at mixtures with different sized inclusions. An in-depth review of these theories is presented in [21].

In addition to dielectric inclusions, metallic inclusions can also be used. It is well known that some metallic materials tend to have very high equivalent permittivity and permeability values over certain frequencies as calculated from the Drude model [22], [23], and as they are typically readily available, heterogeneous structures having high $\varepsilon_{e f f}$ can be obtained using the appropriate nano-engineering fabrication methods. 
Table I: List of parameters and symbols

\begin{tabular}{|c|l|}
\hline$a$ & Radius of spherical inclusions \\
\hline$l$ & Length of cubical inclusions \\
\hline$s$ & Centre-to-centre spacing of inclusions \\
\hline$p$ & Volume fraction \\
\hline$d$ & Sample thickness \\
\hline$\varepsilon_{1}, \mu_{1}$ & Frequency \\
\hline$\varepsilon_{2}, \mu_{2}$ & Inclusion's permittivity and permeability \\
\hline$\varepsilon_{\text {eff }}, \mu_{\text {eff }}$ & Effective permittivity and permeability \\
\hline
\end{tabular}

\section{A. Analytical Results}

Graphical results of examples based on the theories in [6], [10-14], are given in Fig. 2. The following values were chosen to examine the behaviour of the canonical equations: $a=$ $10-85 \mu \mathrm{m}, s=170 \mu \mathrm{m}, \varepsilon_{1}=3.04, \varepsilon_{2}=11.9$, and $\mu_{1}=\mu_{2}=$ 1. The line marked '[10]' shows the equations in [10] should only be applied when the host material is electromagnetically close to free space. The results using the equations from these papers all produce similar results and do not significantly vary over the frequency range considered. As shown in Fig. 2, the effective $\varepsilon$ of the mixture increases with the inclusion's volume ratio, and there are little or no changes, except for [12] in the effective $\mu$.

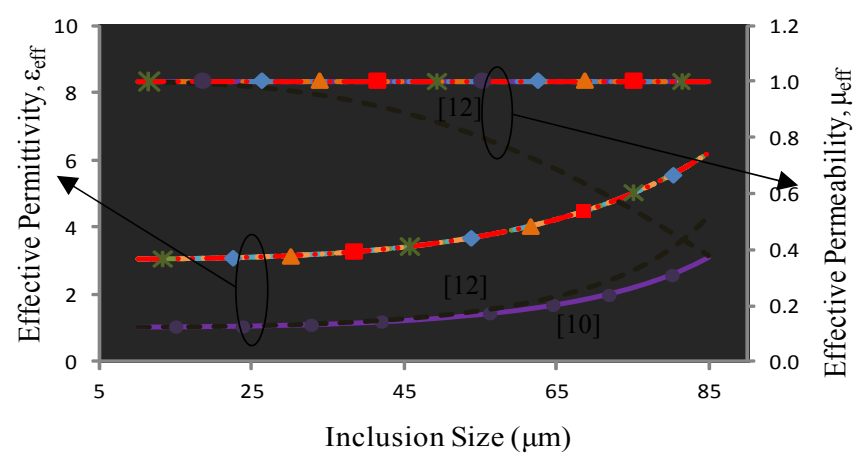

Fig. 2. Variation of effective $\varepsilon$ and $\mu$ of a heterogeneous medium with size, $a$

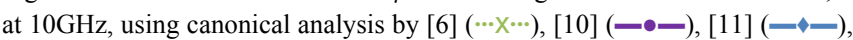
[12] (一 -), [13] (一ㄴ), [14] (一 - $)$.

The equations from [11] are more robust as they use the full scattering equations and account for the physical and EM properties of the materials that constitute the heterogeneous medium and as such might be the best to use for further studies. Note the results from [10] do not include the EM properties of the host and the results from [12] do not include a frequency term and this emphasizes the difference in $\varepsilon_{e f f}$ computed.

\section{Electromagnetic Simulation ANALYSiS}

3-D FDTD (EMPIRE XCcel ${ }^{\mathrm{TM}}$ ) simulations have been used to simulate heterogeneous structure as a comparison with the analytical results and to allow the work to be extended to finite and/or non-uniform structures. The effective $\varepsilon$ and $\mu$ of a medium can be obtained from the S-parameters as shown in [18], [19], [24-28]. The S-parameters obtained were used in a "Resonant Inverse Scattering Formalism" [18] to obtain the effective $\varepsilon$ and $\mu$ of the simulated structures in this paper, which differ from that in [18] which has 2-D disc inclusions.

The structure is set up such that it is infinite perpendicular to the direction of the incident EM plane wave. This allows us to reproduce the boundary conditions for the equations in the literature in two dimensions. The infinite boundary conditions are achieved using perfect electric and magnetic conductors (PEC and PMC) parallel to the orientations of the $\mathrm{E}$ and $\mathrm{H}$ fields. The simulation setup is shown in Fig. 3. The structure is finite along the $\mathrm{X}$ axis and different numbers of layers of spheres are included in this dimension.
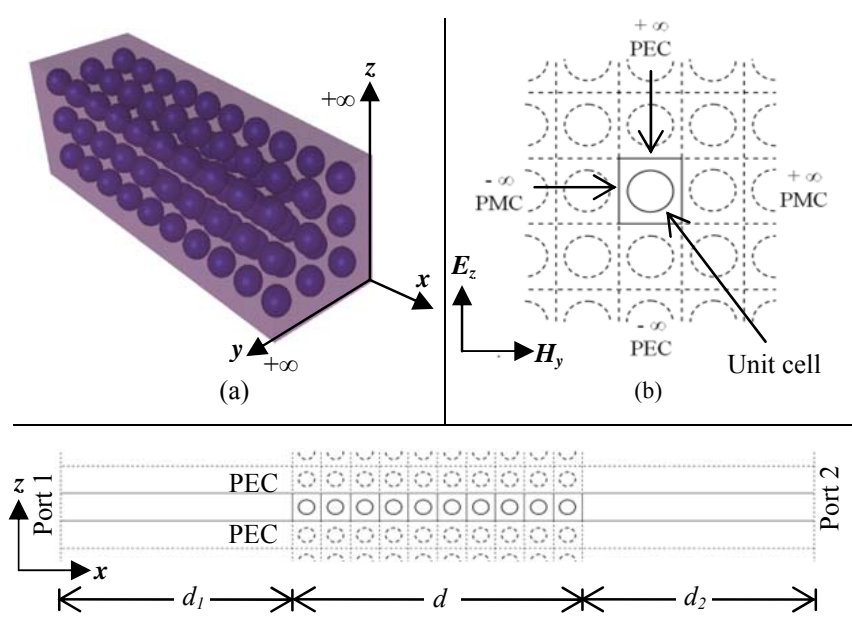

(c)

Fig. 3. (a) 3-D representation of a $3 \times 3$ section of the infinite array, (b) and (c) 2-D views of a 10-layer simulation setup of the heterogeneous structure. The dotted lines represent the planes of symmetry where the structure extends to infinity and are represented by using PECs and PMCs.

The reference planes (where the phases of the S-parameters are calculated) are placed away from the structure under test (or discontinuities) to ensure "higher order evanescent modes" are properly attenuated [27]. This is also to allow enough Yee cells per wavelength and that good meshing resolution (discretization) are used for the structure during simulation. These planes are represented by Port 1 and Port 2 as shown in Fig. 3. PML (Perfectly Matched Layers) boundaries are used at Port 1 and Port 2 to minimize reflections at the ports. The Sparameters from the simulations are fed into the inversion process, which takes into account the distances from the structure to the measurement planes and the thickness of the sample, $d$. The distance from Port 1 to the slab is $d_{1}$, the distance from Port 2 to the other end of the slab is $d_{2}$, as shown in Fig. 3, and the phase of the $S_{11}$ from the simulations is $\varphi_{1}$ and the phase of the $S_{21}$ is $\varphi_{2}$. The appropriate phases required for the inversion process are the S-parameter phases at the measurement planes transferred to the edges of the slab [27], [28]. These corrected phases are given in (2) by:

$$
\begin{aligned}
& \varphi_{1 c}=\varphi_{1}+2 k_{0} d_{1} \text { for the } S_{11}, \text { and } \\
& \varphi_{2 c}=\varphi_{2}+k_{0} d_{2}+k_{0} d_{1} \text { for the } S_{21},
\end{aligned}
$$


where $k_{0}=2 \pi / \lambda$.

First, the refractive index, $n$ and the wave impedance, $\eta$ of the medium are obtained. From this, the effective $\varepsilon$ and $\mu$ of the structure is calculated using equation (3):

$$
\begin{aligned}
& \varepsilon(\omega)=n(\omega) / \eta(\omega) \\
& \mu(\omega)=n(\omega) \cdot \eta(\omega)
\end{aligned}
$$

As an inverse cosine function is involved in obtaining the refractive index of the medium, discontinuities appear in the phase of the medium, $\Delta \phi_{d}$. But since the refractive index is required to be continuous over frequency for accurate results, a "rectification algorithm" (see Appendix I) is needed [18] to provide a continuous phase, see Fig. 4. Without this rectification, $n$ follows the discontinuity in the phase, falling to zero at the frequencies of the phase minima and peaking at the frequencies of the phase maxima. Thus, $n$ is only accurate at frequencies below the first discontinuity (see Fig. 5) without the rectification algorithm.

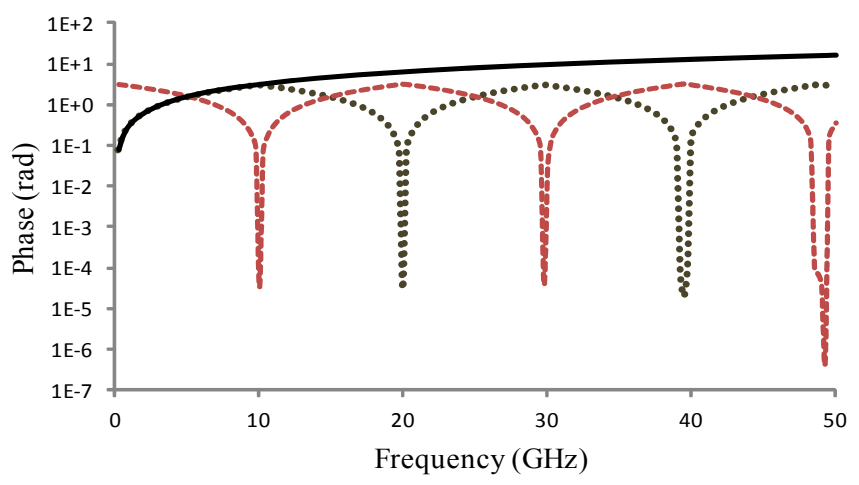

Fig. 4. An example of the discontinuous phase $\Delta \varphi_{d}(\cdots \cdots), \pi-\Delta \varphi_{d}(----)$ and rectified phase $\Delta \varphi_{r}(-)$.

Fig. 4 shows a plot of these phases. The line labelled $\left(\pi-\Delta \phi_{d}\right)$ helps to highlight these discontinuities. Since the refractive index is subject to the rectification process, a relatively continuous plot can be achieved. However, as the wave impedance is still a direct function of the S-parameters, its behaviour still depends on $S_{11}$ and $S_{21}$, hence the 'spikes' in its plots will appear in the effective $\varepsilon$ and $\mu$ graphs.

To illustrate the effect of the rectification algorithm on the accuracy and continuity of the inversion results, $\varepsilon_{e f f}$ and $\mu_{e f f}$, Fig. 5 shows the obtained $\varepsilon_{e f f}$ with and without the algorithm.

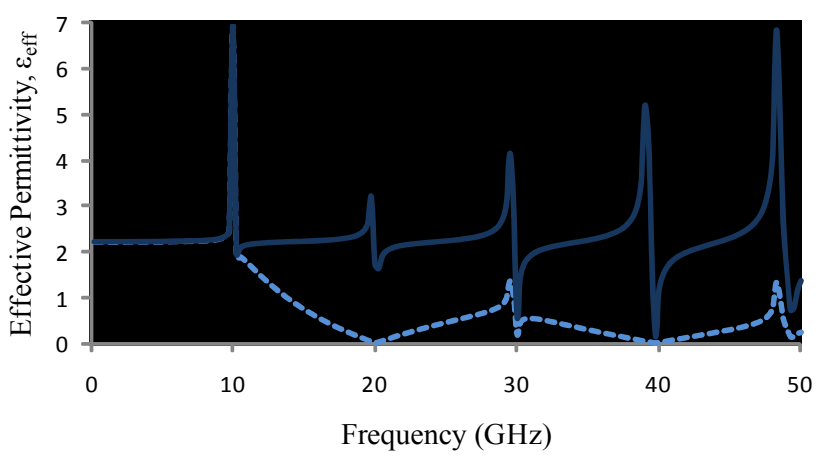

Fig. 5. An example of the variation of $\varepsilon_{\text {eff }}$ with frequency with (-) and without (---) rectification of the phase of the medium.
It is worth noting that information from the simulation is not lost by using the rectification process. The group delay of the medium before and after the inversion process (including rectification) remains the same (results are not shown for space reasons).

At certain frequencies related to the thickness of a dielectric slab and its EM properties, such that $d=n \lambda_{g} / 2$ where $\lambda_{g}$ is the guided wavelength, and $n$, an integer, $=1,2,3 \ldots$, the slab is transparent to an incident plane wave [28]. Therefore the $S_{11}$ values are close to zero $\left(\operatorname{Re}\left(S_{11}\right), \operatorname{Im}\left(S_{11}\right) \cong 0\right)$, while $S_{21} \cong 1 \quad\left(\operatorname{Re}\left(S_{21}\right) \cong 1, \operatorname{Im}\left(S_{21}\right) \cong 0\right)$. This explains the "spike" points (shown for the plots in Fig. 5 and other $\varepsilon_{e f f}$ plots) also known as the half-wavelength impedance matching frequencies [28], [29] or thickness resonance points [30].

\section{Results}

\section{A. Validation of Inversion Process with Homogenous Slab}

Several results from simulations of media with known EM parameters were used to test the inversion process. Fig. 6(a) shows the 3D simulation setup. The green (top figure - - ) and the red (bottom figure - - ) horizontal lines in Fig. 6(b) represent the PMC $\left(y_{\min }, y_{\max }\right)$ and PEC $\left(z_{\min }, z_{\max }\right)$ boundary conditions respectively.

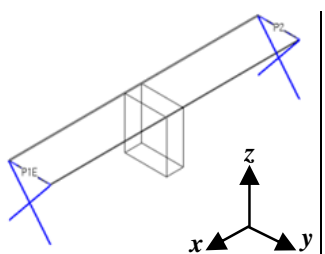

(a)

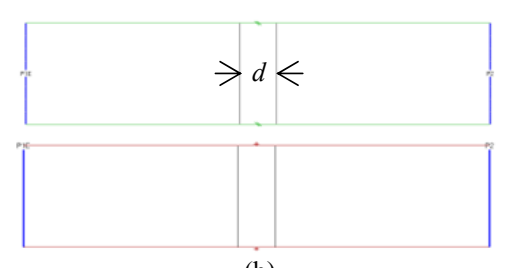

(b)
Fig. 6. (a) $3 \mathrm{D}$ and (b) $2 \mathrm{D}$ representations of the simulation setup for a homogenous slab in EMPIRE XCcel ${ }^{\mathrm{TM}}$.

In order to validate the process, a homogenous slab of known EM properties was simulated and the S-parameters put through the inversion process. The real and imaginary parts of the S-parameters are shown in Fig. 7. Data used: $d=10 \mathrm{~mm}$, $d_{1}=d_{2}=69.5 \mathrm{~mm}$ and $\varepsilon_{\text {slab }}=2.2$.

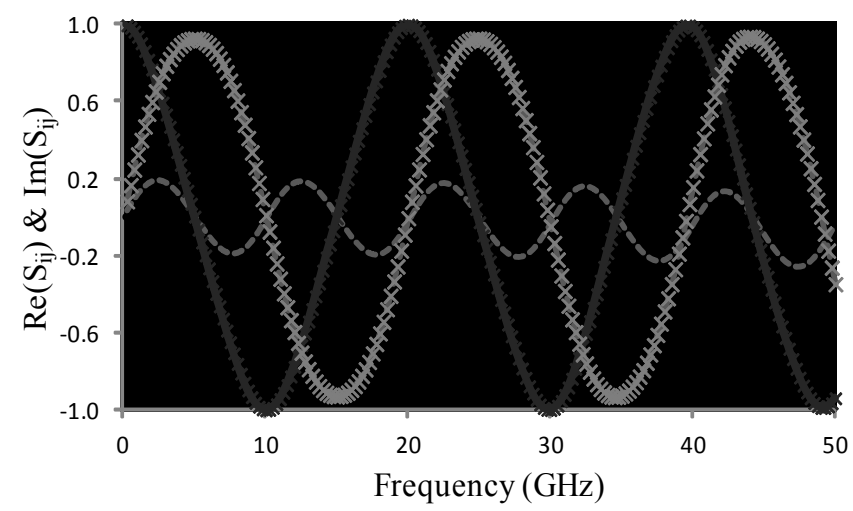

Fig. 7. Real (continuous black) and imaginary (dashed grey) parts of (a) $S_{11}$ (plain) and (b) $S_{21}$ (starred). 


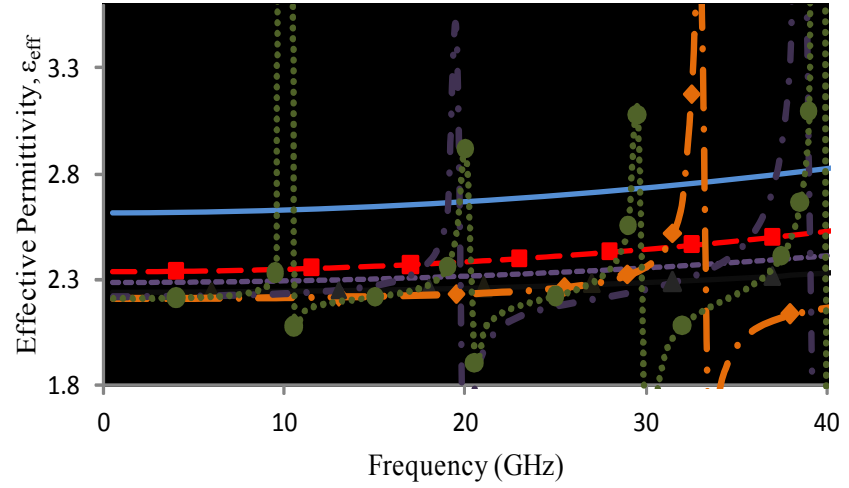

Fig. 8. Variation with frequency of the permittivity derived via the inversion process of a $0.1 \mathrm{~mm}(-), 0.3 \mathrm{~mm}(-\mathbf{-}), 0.5 \mathrm{~mm}(--), 1 \mathrm{~mm}(-\boldsymbol{\Delta}-), 3$ $\mathrm{mm}(-\bullet), 5 \mathrm{~mm}(-\cdots)$ and $10 \mathrm{~mm}(\cdots \bullet \cdots)$ homogeneous slabs with $\varepsilon_{r}=$ 2.2 .

The first thickness resonance for the $1 \mathrm{~mm}$ slab occurs at approximately $100 \mathrm{GHz}$. The first resonance occurs at the following frequencies for different dielectric thicknesses: 1 $\mathrm{mm}$ at $81 \mathrm{GHz}, 5 \mathrm{~mm}$ at $19.5 \mathrm{GHz}$, and $10 \mathrm{~mm}$ at $10 \mathrm{GHz}$. The result from the $0.1 \mathrm{~mm}$ slab is inaccurate as it is higher than the known value. Thus, an appropriate slab thickness has to be used for simulations in order to avoid "measurement uncertainties" [29] due to thin samples and obtain accurate results from the inversion process. Where the thickness is greater than $0.3 \mathrm{~mm}$, the value of $\varepsilon_{e f f}$ is approximately the known value, 2.2, and is relatively stable below the first resonant frequency. Therefore, the inversion process has been validated with a homogeneous sample. Above certain frequencies, the real part of the refractive index decreases with increasing frequency (not shown for space reasons) which may be due to anomalous dispersion [31] or because the inversion solution is not well-behaved over these frequencies.

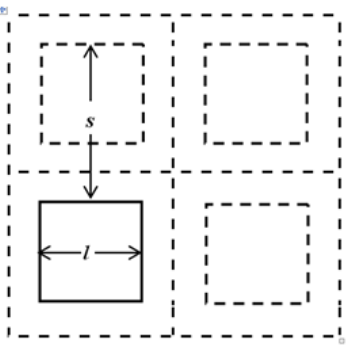

(a)

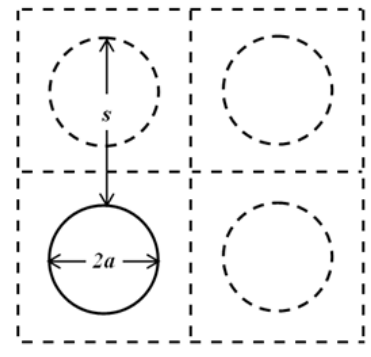

(b)
Fig. 9. Physical geometry of (a) cubical and (b) spherical inclusions

\section{B. Heterogeneous Structures with Cubic Inclusions}

Initially, cubes (see Fig. 9 (a)) were used for simulations as they had less demand for computing resources and avoided the stair-casing effect that curved surfaces are subject to when meshed in FDTD simulations. Spheres will be used later (Section IV C) to compare with results from the canonical equations. 60 layers of inclusions in the direction of wave propagation were used in the simulations. However, fewer layers were found to produce similar results as the number of layers increases, the results tend to the same values.
Data used in Fig. 10: cube length, $l=150 \mu \mathrm{m}, s=250 \mu \mathrm{m}$, $\varepsilon_{1}=3.55, f=1-30 \mathrm{GHz}$ and $\varepsilon_{2}=11.9$. A $150 \mu \mathrm{m}$ cube has approximately the same volume as a $93.05 \mu \mathrm{m}$ radius sphere which was used in the canonical equations as a comparison.

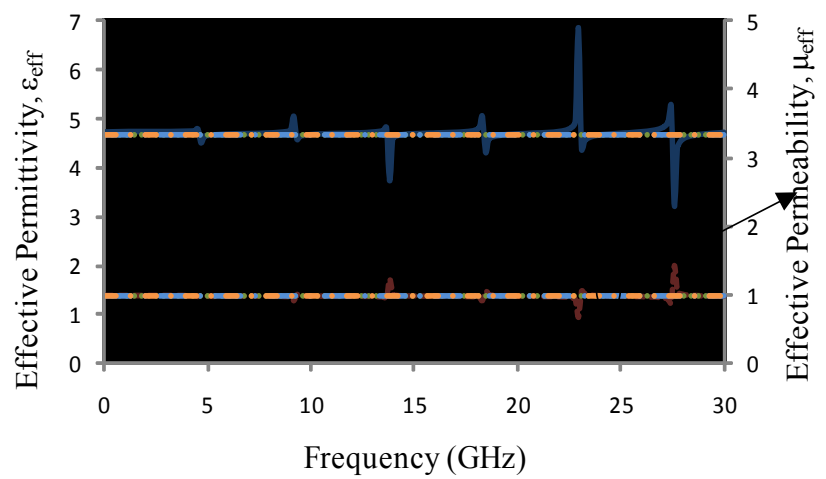

Fig. 10. Variation with frequency of effective $\varepsilon(-)$ and $\mu(--)$ using FDTD simulations and canonical equations [6] (*.), [11] (-· -,$[14](-\cdot-)$, of a heterogeneous structure. The FDTD results use equally spaced cubic inclusions while the canonical results use spheres with the equivalent volume.

An equivalent volume process was used, that is:

Volume of a sphere, $4 \pi r^{3} / 3=$ Volume of a cube, $l^{3}$

The EM simulation gives an average (over the frequencies examined) $\varepsilon_{e f f}=4.67$, while the results from the canonical equations show excellent agreement with an $\varepsilon_{e f f}=4.7$ as averaged from [6], [11], [14].

\section{Heterogeneous Structures with Spherical Inclusions}

To see how the simulated values of effective $\varepsilon$ and $\mu$ compare with the canonical equations, $150 \mu \mathrm{m}$ diameter spheres were used in the FDTD simulations. (Fig. 9 (b)). The spacing, $s, \varepsilon_{1}$, and $\varepsilon_{2}$ are the same as in Section B. The results shown in Fig. 11 have an average $\varepsilon_{e f f}=4.11$ from the theoretical equations and an $\varepsilon_{e f f}=4.11$ from the simulations. For the same spacing, the $150 \mu \mathrm{m}$ cubes have a larger volume fraction than the $150 \mu \mathrm{m}$ spheres and therefore have a higher effective permittivity.

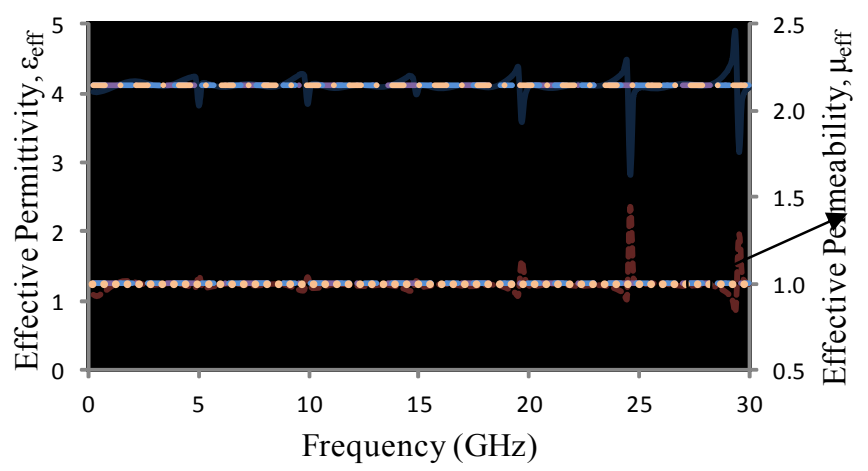

Fig. 11. Variation with frequency of effective $\varepsilon(-)$ and $\mu(--)$ using

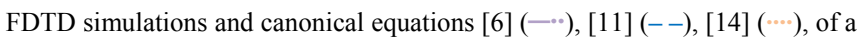
heterogeneous structure with equally spaced $150 \mu \mathrm{m}$ spheres.

The results in Fig. 10 and 11 show there is good agreement (thus the severe overlap) between the theoretical equations and 
the inversion via simulations results allowing us to use the $3 \mathrm{D}$ simulation techniques to design and control the permittivity of heterogeneous substrates. For speed but with lesser accuracy, cubes can be used to represent spherical inclusions in the simulations to get a reasonable estimate of the effective EM properties of the heterogeneous medium. For heterogeneous media in which $\varepsilon_{2}>\varepsilon_{1}$, the $\varepsilon_{\text {eff }}>\varepsilon_{1}$.

\section{Parametric Studies of Heterogeneous Structures}

For comparison with the canonical formulations, parametric studies were carried out on (i) the spherical inclusions' permittivity, (ii) the volume fraction of the inclusions and (iii) the number of layers.

\section{Inclusion Permittivity, $\varepsilon_{2}$}

Data used in Fig. 12: $a=80 \mu \mathrm{m}, s=170 \mu \mathrm{m}$, and host permittivity, $\varepsilon_{1}=2.1$. The results of the effective permittivity while varying only $\varepsilon_{2}$, from the EM simulations is shown in Fig. 12(a) and that from the canonical equations in Fig. 12(b).

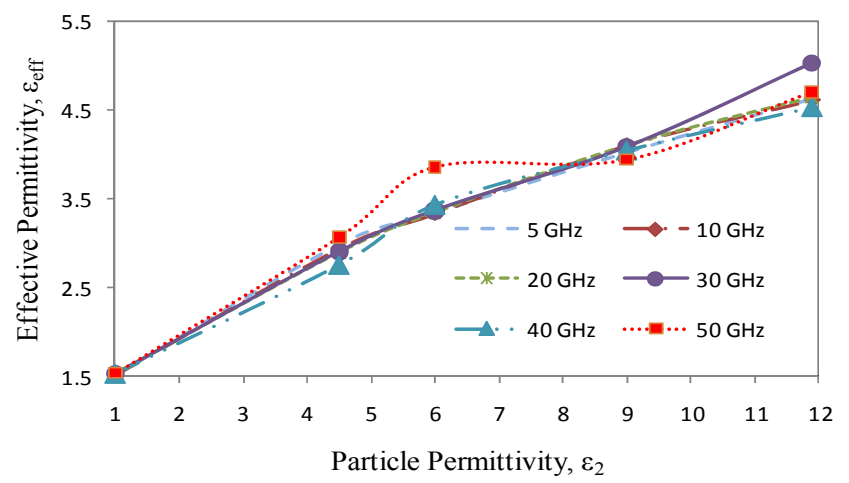

(a)

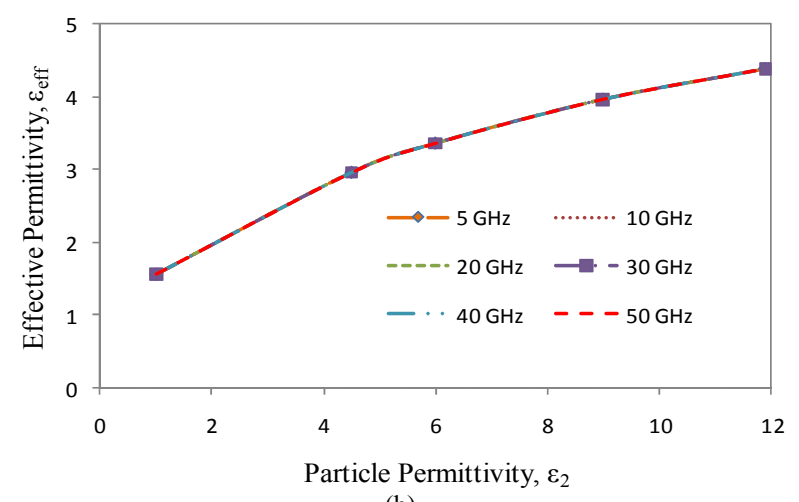

(b)

Fig. 12. Variation of effective $\varepsilon$ with inclusion permittivity, $\varepsilon_{2}$, of a heterogeneous structure from (a) simulations via inversion and (b) canonical equations at different frequencies (average results from [6], [11], [14]).

When these graphs are plotted on the same scale, they overlap showing further that the parametric study on $\varepsilon_{2}$ via simulations and equations agree. This near-linear variation of effective permittivity with inclusion permittivity shows that as $\varepsilon_{2}$ increases, $\varepsilon_{\text {eff }}$ increases at a similar rate.

\section{Inclusion Volume Fraction, $p$}

Data used in Fig. 13: $\varepsilon_{1}=3.55, \varepsilon_{2}=11.9$ and $s=250 \mu \mathrm{m}$
For Fig. 13 (b), $\mu_{1}=\mu_{2}=1$. The sphere size was increased to vary the inclusions' volume fraction.

Except for the two points in $15 \mathrm{GHz}$ and $30 \mathrm{GHz}$ plots in Fig. 13(a), the results from the simulation and canonical equations agree to great extent, causing these lines to overlap.

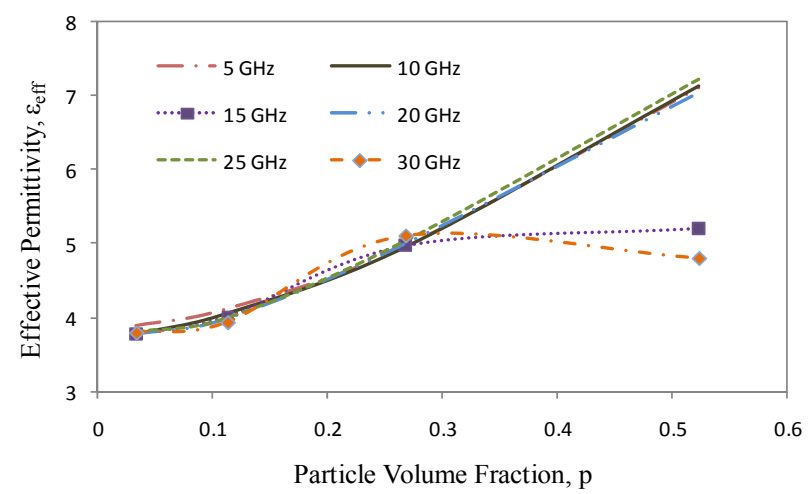

(a)

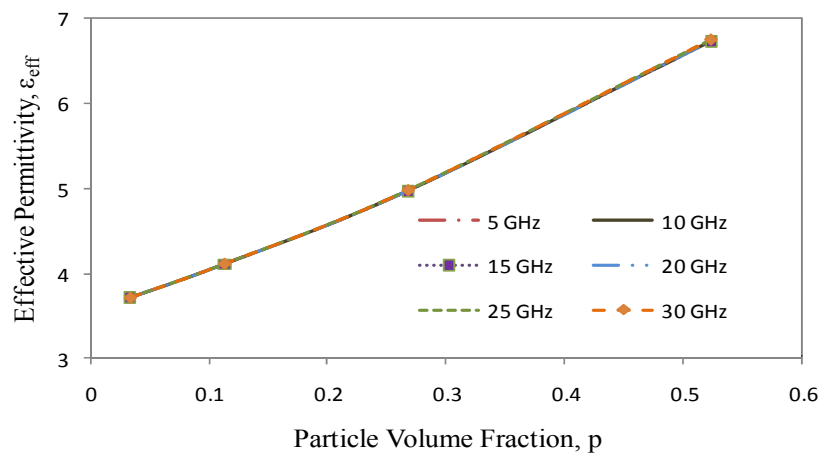

(b)

Fig. 13. Variation of effective $\varepsilon$ with inclusion's volume fraction, $p$, of a heterogeneous structure, from (a) simulations via inversion and (b) canonical equations at different frequencies (average results from [6], [11], [14]).

The two deviating points are for when the volume fraction, $p=0.524$, and that is when the spheres are in physical contact $(a=125 \mu \mathrm{m})$. And at these frequencies $(15$ and $30 \mathrm{GHz})$ the structure is completely transparent to the incoming wave and so $S_{21} \approx 1$, and $S_{11} \approx 0$, which results in numerical errors.

Even though the total thickness of the sample, the host and inclusions' permittivity and permeability were the same for all the volume fractions simulated, the frequencies at which the sample was transparent to the wave (where the spikes occurred) differed for each sphere size because the volume fraction changes with size.

\section{Number of layers}

The effect of the number of layers of spheres used in the simulations in the axis of finite thickness is shown in Fig. 14. Data used: $a=75 \mu \mathrm{m}, s=250 \mu \mathrm{m}, f=1-30 \mathrm{GHz}, \varepsilon_{1}=3.55$ and $\varepsilon_{2}=11.9$. 


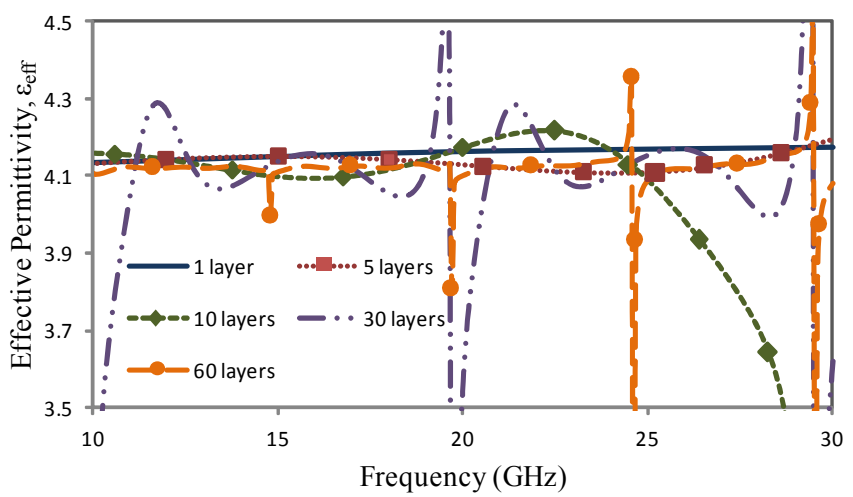

Fig. 14. Variation of calculated effective $\varepsilon$ with frequency, $f$, of a simulated heterogeneous structure for different number of layers of spheres.

From Fig. 14, it can be seen that for the different number of layers, the effective permittivity is on average the same, but the spikes shown occur at a lower frequency as the number of layers increases. There are no spikes for the 1-layer and 5layer media within the considered frequency range. For this specific geometry, the 1-layer structure produces reasonable results.

As nano-fabrication facilities are currently unavailable to us, we have used scaled measurements using a $0.75-1.1 \mathrm{GHz}$ waveguide. $10 \mathrm{~mm}$ chrome steel ball bearings spaced $13 \mathrm{~mm}$ apart were placed in a Rohacell ${ }^{\circledR}$ host. These sizes are scaledup values of micro-sized inclusions and spacings. Simulating this structure at $1 \mathrm{GHz}$ gave an $\varepsilon_{e f f}$ of 2.09 , and $\mu_{\text {eff }}=0.80$, while the measurements gave $\varepsilon_{\text {eff }}=1.83$, and $\mu_{\text {eff }}=0.76$. These values show good agreement and corroborate our canonical and simulation analysis.

\section{A Patch Antenna with a Heterogeneous SubStrate}

For further confirmation of the results obtained from the canonical equations and the simulation (via the inversion process), a patch antenna was used. This also extends the results from an infinite medium to a finite medium. A patch antenna was placed on a heterogeneous substrate containing spherical inclusions and also on a homogenous substrate with the same permittivity as calculated from the FDTD and inversion process. The $S_{11}$ results from the two simulations were then compared. Note, the use of substrates with microsized inclusions will not inherently improve the efficiency of the antenna. However, the micro-sized inclusions allow the effective permittivity to be controlled on a fine scale. Therefore, patch antennas can be designed in future where the permittivity can be varied smoothly across the substrate and also as a function of the height of the substrate. This variation can then be exploited to improve the efficiency and bandwidth as in textured dielectrics.

The cross-section of the patch on the heterogeneous substrate is shown in Fig. 15. Data used: $a=75 \mu \mathrm{m}, s=$ $250 \mu \mathrm{m}, f=1-30 \mathrm{GHz}, \varepsilon_{1}=3.55, \varepsilon_{2}=11.9$, patch size $=3$ $\mathrm{mm}$ by $5 \mathrm{~mm}$ by $30 \mu \mathrm{m}$, and substrate size $=12 \mathrm{~mm}$ by $12 \mathrm{~mm}$ by $1 \mathrm{~mm}$.

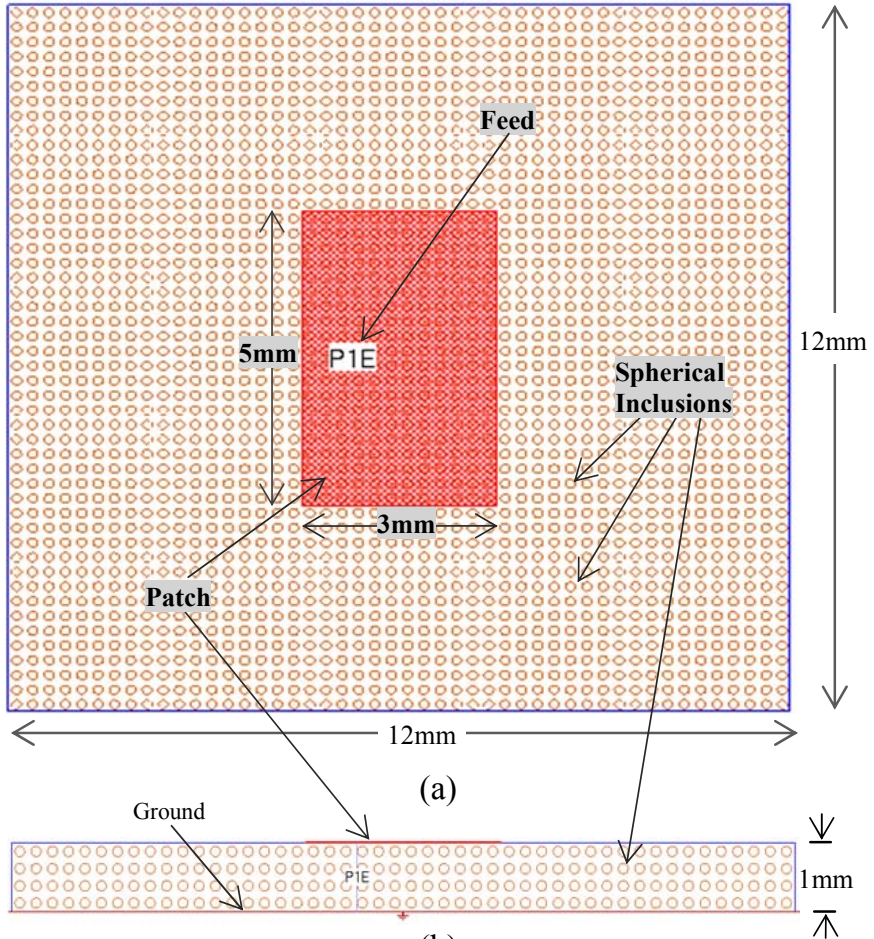

(b)

Fig. 15. (a) Top and (b) side views of a patch antenna on heterogeneous substrate. P1E represents the port.

These values of EM properties and geometrical dimensions of the semi-infinite heterogeneous medium in the preceding sections (Section IV C) gave an $\varepsilon_{\text {eff }}$ of 4.11 and this value was used for the homogeneous substrate. The $S_{11}$ results for patch on the heterogeneous and homogenous substrates are shown in Fig. 16.

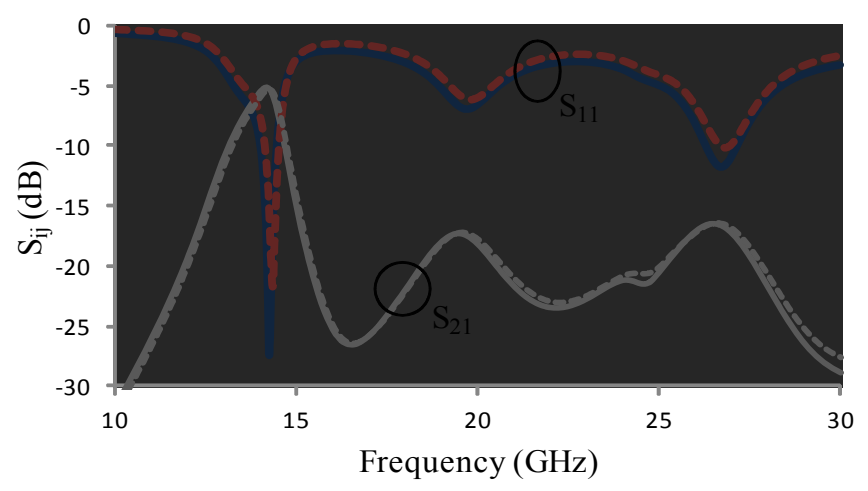

Fig. 16. Variation of return loss $S_{11}$ (thick lines) with frequency for a patch antenna on homogeneous (continuous lines) and heterogeneous (dashed lines) substrates. $\mathrm{S}_{21}$ (thin lines) shows the mutual coupling of two adjacent patches printed on each substrate.

Simulations of two patches separated by $2 \mathrm{~mm}$ were carried out in order to show that the coupling between the patches was consistent in both substrates. The S-parameters for coplanar patch antennas on the heterogeneous and homogenous substrates are shown in Fig. 16 which shows that the mutual coupling between the antennas agrees to a great extent on the 
two substrates.

The close agreement between the homogenous and heterogeneous plots provides further confidence that the inversion process is accurate and that these heterogeneous materials can be adequately used in finite antenna designs. Including a loss tangent of 0.0027 and 0.01 for the host and inclusions respectively, yielded an antenna efficiency of $85 \%$ which is on a par with the efficiency of the patch on the homogenous substrate with a loss tangent of 0.003 . The patch on the heterogeneous medium takes about 20 hours to run as there are many Yee cells in both the $\mathrm{X}$ and $\mathrm{Y}$ directions.

\section{CONCLUSIONS}

It is possible to increase the effective permittivity of a host material with dielectric inclusions. The results from the analytical equations verified the simulation results and the inversion process. The analytical equations use spherical inclusions but this paper has shown that the inclusions can be approximated as cubes with the same equivalent volume. The inversion process produces spikes (thickness resonances) at frequencies where there is near total transmission - these spikes are numerical errors and do not affect the actual permittivity. The original theoretical work was based on infinite media; however, this paper has shown that the results are valid for finite structures, such as patch antennas.

These structures take a considerable amount of simulation time, (at least 2 hours) depending on geometry and materials. With the ever improving performance of simulation packages, fine scale structures detailed in this paper can be computed in manageable time scales. Note, that once the effective permittivity is known, the antenna design duration can be reduced by approximating the heterogeneous structures with the equivalent homogeneous material for some of the simulation runs.

It should be noted that higher ratios of the effective permittivity to the host permittivity can be achieved with different host and inclusions permittivities as well as with different volume fractions.

\section{APPENDIX I}

This section lists the main equations in the inversion process and explains the phase rectification part of the process [18] see Section III. Note: this appendix includes details not included in [18].

$$
\eta=\frac{1+A}{1-A}= \pm \sqrt{\frac{V+1}{V-1}}
$$

where $A=V \pm \sqrt{V^{2}-1}$

$$
\operatorname{Re}\{n\}=\frac{\cos ^{-1}(\operatorname{Re}\{x\} /|x|)}{k_{0} d} ; \quad \operatorname{Im}\{n\}=-\frac{\ln |x|}{k_{0} d}
$$

where

$$
\begin{gathered}
V=\frac{1+S_{11}^{2}-S_{21}^{2}}{2 S_{11}} ; \quad x=\frac{S}{1+R-A S R} ; \quad S=S_{11}+S_{21} ; \quad R=\frac{S_{11}}{S_{21}} \\
\Delta \phi_{d}=k_{0} d \operatorname{Re}\{n\}=\cos ^{-1}(\operatorname{Re}\{x\} /|x|)
\end{gathered}
$$

To rectify the phase, the first increasing function, $\Delta \phi_{d}$ or $\left(\pi-\Delta \phi_{d}\right)$ is equal to the rectified phase, $\Delta \phi_{r}$, at frequencies below that of the first discontinuity, say, at $f_{1}$.

At $f_{1}$, the second function which is now increasing is added to the first from $f_{1}$ to the next discontinuity, say $f_{2}$. Thus,

$$
\begin{aligned}
\Delta \phi_{r}= & \Delta \phi_{d}\left(f_{1}\right)+\left(\pi-\Delta \phi_{d}\right) \\
& \text { or }\left(\pi-\Delta \phi_{d}\right)\left(f_{1}\right)+\Delta \phi_{d}
\end{aligned}
$$

At $f_{2}$, when the second function starts decreasing, the first function is increasing and is added from $f_{2}$ to the next discontinuity, say $f_{3}$,

$$
\begin{aligned}
& \Delta \phi_{r}=\Delta \phi_{d}\left(f_{1}\right)+\left(\pi-\Delta \phi_{d}\right)\left(f_{2}\right)+\Delta \phi_{d} \\
& \quad \text { or }\left(\pi-\Delta \phi_{d}\right)\left(f_{1}\right)+\Delta \phi_{d}\left(f_{2}\right)+\left(\pi-\Delta \phi_{d}\right)
\end{aligned}
$$

This process is continued until the upper end of the frequency range. The rectified phase, $\Delta \phi_{r}$ is now a continuous function.

$\Delta \phi_{r}$ is used to re-compute $\operatorname{Re}\{n\}$ in (A2), that is,

$$
\operatorname{Re}\{n\}=\frac{\Delta \phi_{r}}{k_{0} d}
$$

$\varepsilon$ and $\mu$ are then calculated using (2).

\section{ACKNOWLEDGEMENTS}

The authors wish to thank Dr. Jon Rigelsford and Dr. Lee Ford of The University of Sheffield, UK for their help with the measurements.

\section{REFERENCES}

[1] YouTube Video - "IBM Self Assembly Technology Creates Airgap Microprocessors.” (2007, May 3). Available: http://www.youtube.com/watch?v=T8p8 zZNJEU

[2] YouTube Video - "Nano, the next dimension (Film produced for European Commission).” (2008, March 9). Available: http://www.youtube.com/watch?v=eCpkq AeX50

[3] F. Juillerat, H. H. Solak, P. Bowen, and H. Hofmann, "Fabrication of large-area ordered arrays of nanoparticles on patterned substrates", Inst. Of Phys. Pub., Nanotech., vol. 16, no. 8, pp.1311-1316, June 2005.

[4] C. Hsu, and Y. Lee, "Fabrication of flexible nano-wired polarizer by contact-transferred and mask embedded lithography and polyurethane acrylate method", Proc. $5^{\text {th }}$ IEEE Int. Conf. NEMS, Xiamen, China, 2010, pp. 893-897.

[5] Y. H. Seo, D. Choi, and K. Whang, "A new fabrication method of nanofluidic filters using self-assembly of nano-spheres and surface tension", $19^{\text {th }}$ Proc. IEEE Int. Conf. MEMS, Istanbul, 2006, pp.306-309.

[6] L. Lewin, "The electrical constants of a material loaded with spherical particles”, IEE-Part III: Radio Comm. Eng., vol. 94, issue 27, pp. 65-68, Jan. 1947.

[7] L. Rayleigh, "On the influence of obstacles arranged in rectangular order on the properties of a medium", Phil. Mag., series 5, vol. 34, pp. 481502, 1892.

[8] J. Brown, "Artificial dielectrics having refractive index less than unity", Proc. IEE-Part IV: Inst. Mono., vol. 100, issue 5, pp. 51-62, Sept. 1953.

[9] W. T. Doyle, "The Clausius-Mossotti problem for cubic array of spheres", J. App. Phys., vol. 49, issue 2, pp. 795-797, Feb. 1978.

[10] X. Cai, R. Zhu, and G. Hu, "Experimental study for metamaterials based on dielectric resonators and wire frame", Metamaterials, vol. 2, issue 4, pp. 220-226, Sept. 2008.

[11] W. T. Doyle, "Optical properties of a suspension of metal spheres", Phy. Rev. B, vol. 39, issue 14, pp. 9852-9858, May 1989.

[12] R. E. Collin, Field Theory of Guided Waves, IEEE Press: NY, 1991, ch. 12.

[13] A. Sihvola, Electromagnetic Mixing Formulas and Applications, IEE Elect. Waves Series 47, 1999, ch. 3. 
[14] I. A. Kolmakov, L. Jylha, S. A. Tretyakov, and S. Maslovki "Lattice of dielectric particles with double negative response", $28^{\text {th }}$ Gen. Ass. Int. Union Radio Sci. (URSI), paper BCD.5 (0109), New Delhi, India, 2005.

[15] C. L. Holloway, E. F. Kuester, J. B-Jarvis, and P. Kabos, "A double negative (DNG) composite medium composed of magnetodielectric spherical particles embedded in a matrix", IEEE Trans. Antennas Propagat., vol. 51, no. 10, pp. 2596-2603, Oct. 2003.

[16] M. I. Kitra, C. J. Panagamuwa, P. McEvoy, J. C. Vardaxoglou, and J. R. James, "Low SAR ferrite handset antenna design", IEEE Trans. Antennas Propagat., vol. 55, issue 4, pp. 1155-1164, April 2007.

[17] G. Kiziltas, D. Psychoudakis, J. L. Volakis, and N. Kikuchi, "Topology design optimization of dielectric substrates for bandwidth improvement of a patch antenna", IEEE Trans. Antennas Propagat., vol. 51, issue 10, pp. 2732-2743, Oct. 2003.

[18] N. G. Alexopoulos, C. A. Kyriazidou, and H. F. Contopanagos, "Effective parameters for metamorphic materials and metamaterials through a resonant inverse scattering approach", IEEE Trans. Microw. Theory Tech., vol. 55, no. 2, pp. 254-267, Feb. 2007.

[19] I. Awai, Y. Maegawa, and T. Ishizaki, "Measurement of effective material constants of artificial dielectrics made of spherical metal particles", Asia Pacific Microw. Conf. 2009, Singapore, 2010, pp. 16551658.

[20] J. A. Stratton, Electromagnetic Theory, New York: McGraw-Hill Book Inc., 1941, ch. 9.

[21] C. C. Njoku, W. G. Whittow, and J. C. Vardaxoglou, "Comparative study of nanomaterials' effective properties using canonical equations", Proc. $6^{\text {th }}$ Annu. Lough. Antennas. Propagat. Conf., Loughborough, UK, 2010, pp. 413-416.

[22] M. A. Ordal, et al., "Optical properties of the metals $\mathrm{Al}, \mathrm{Co}, \mathrm{Cu}, \mathrm{Au}, \mathrm{Fe}$, $\mathrm{Pb}, \mathrm{Ni}, \mathrm{Pd}, \mathrm{Pt}, \mathrm{Ag}, \mathrm{Ti}$, and $\mathrm{W}$ in the infrared and far infrared", Applied Optics, vol. 22, no. 7, April 1983.

[23] C. A. Balanis, Advanced Engineering Electromagnetics. New York: Chichester: Wiley, 1989, ch. 2 .

[24] C. C. Njoku, W. G. Whittow, and J. C. Vardaxoglou, "Study on the variation in dielectric properties of heterogeneous substrates composed of nanomaterials", Proc. $5^{\text {th }}$ Annu. Eur. Conf. Antennas Propagat., Rome, Italy, 2011, pp. 488-492.

[25] K. K. Karkkaninen, A. H. Sihvola, and K. I. Nikoskinen, "Effective permittivity of mixtures: Numerical validation by the FDTD method", IEEE Trans. Geos. Remote Sensing, vol. 38, no. 3, pp. 1303-1308, May 2000.

[26] D. R. Smith, D. C. Vier, T. Koschny, and C. M. Soukoulis, "Electromagnetic parameter retrieval from inhomogeneous metamaterials", Phys. Rev. E71, 036617, 2005.

[27] W. B. Weir, "Automatic measurement of complex dielectric constant and permeability at microwave frequencies", Proc. IEEE, vol. 62, no. 1, pp. 33-36, Jan. 1974.

[28] N. Ida, Engineering Electromagnetics. New York: Springer Verlag, 2004, ch. 13.

[29] J. B. Jarvis, E. J. Vanzura, and W. A. Kissick, "Improved technique for determining complex permittivity with the transmission/reflection method", IEEE Trans. Microw. Theory Tech., vol. 38, no. 8, pp. 10961103, Aug. 1990.

[30] A. H. Boughriet, C. Legrand, and A. Chapoton, "Noniterative stable transmission/reflection method for low-loss material complex permittivity determination", IEEE Trans. Microw. Theory Tech., vol. 45, no. 1, pp. 52-57, Jan. 1997.

[31] W. West, “Absorption of electromagnetic radiation", in AccessScience, CMcGraw-Hill Companies, 2008. Available:

http://www.accessscience.com

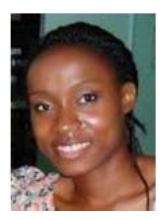

Chinwe C. Njoku received the B.Sc. in electrical/electronics engineering from The University of Lagos, Nigeria in 2006 and her M.Sc. from Loughborough University, U.K in 2009. She is currently pursuing her Ph.D. on heterogeneous substrates for antenna systems in the School of Electronic, Electrical and Systems Engineering at Loughborough University, UK.

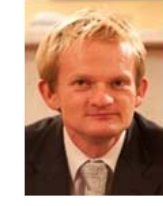

Dr William G. Whittow received the B.Sc. in physics from The University of Sheffield, Sheffield, U.K in 2000 and his Ph.D. in the EEE Department also at Sheffield in 2004. He is currently employed as a Research Associate in the School of Electronic, Electrical and Systems Engineering at Loughborough University, UK. He has published over 60 peer reviewed journal and conference papers in topics related to electromagnetic materials, wearable antennas, VHF antennas, Specific Absorption Rate, FDTD, bioelectromagnetics, phantoms and Genetic Algorithms. Since 2007, he has been the Coordinating Chair of the Loughborough Antennas \& Propagation Conference (LAPC).

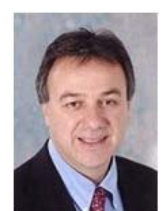

Professor J(Yiannis) C. Vardaxoglou completed his B.Sc. in mathematical physics (1982) and his Ph.D. in Electronics (1985) at the University of Kent, UK. He joined Loughborough University as a lecturer in 1988 and was promoted to Senior Lecturer in 1992 and Professor or Wireless Communications in 1998. He is currently Dean of the School of Electronic, Electrical and Systems Engineering at Loughborough University. He established the Wireless Communications Research (WiCR) group at Loughborough University and heads the Centre for Mobile Communications Research. He has pioneered research, design and development of frequency selective surfaces (FSS) for communication systems, Metamaterials and low SAR antennas for mobile telephony and has commercially exploited a number of his innovations. He has served as a consultant to various industries, holds 6 patents and is the Technical Director of Antrum Ltd (a University spinout company). He has attracted research funding from industry and has been awarded 18 EPSRC research grants. He has published over 160 refereed journals and conference proceeding papers and has written a book on FSS. He was Chairman of the Executive Committee of the IET's Antennas and Propagation Professional Network in the UK and chaired the IEEE's distinguish lecturer program of the Antennas and Propagation Society (APS) for 5 years. He was the General Chair of EuCAP'2007. He has chaired numerous IEE//IET events and has been on the Steering Committee of the European Conference on Antennas and Propagation, EuCAP since 2006. He founded the Loughborough Antennas \& Propagation Conference (LAPC), which has been running since 2005. He was elected a Fellow of the Royal Academy of Engineers in 2011. 